\title{
VARIABLE SOBOLEV CAPACITY AND THE ASSUMPTIONS ON THE EXPONENT
}

\author{
PETTERI HARJULEHTO, PETER HÄSTÖ, \\ MIKA KOSKENOJA and SUSANNA VARONEN \\ Department of Mathematics and Statistics, P.O. Box 68 (Gustaf Hällströmin katu 2b) \\ FIN-00014 University of Helsinki, Finland \\ E-mails: petteri.harjulehto@helsinki.fi, peter.hasto@helsinki.fi, \\ mika.koskenoja@helsinki.fi, susanna.varonen@helsinki.fi \\ Research group home-page: http://mathstat.helsinki.fi/analysis/varsobgroup
}

\begin{abstract}
In a recent article the authors showed that it is possible to define a Sobolev capacity in variable exponent Sobolev space. However, this set function was shown to be a Choquet capacity only under certain assumptions on the variable exponent. In this article we relax these assumptions.
\end{abstract}

1. Introduction. In the early 90's Kováčik and Rákosník [17] introduced variable exponent Lebesgue and Sobolev spaces. In fact, generalized Lebesgue and Sobolev spaces are special cases of so-called Orlicz-Musielak spaces, and in this form their investigation goes back a bit further, to Orlicz [20], Hudzik [15], and Musielak [18], see also Sharapudinov [23]. During the last couple of years Lebesgue and Sobolev spaces with variable exponent have been studied at an increasing pace by Diening [4, 5], Edmunds and Rákosník [6, 7], Fan, Shen and Zhao [9, 10], Cruz-Uribe, Fiorenze and Neugebauer [3], Kokilasvili and Samko [16], and Nekvinda [19], among others.

One area where these spaces have found applications is the study of electrorheological fluids, as described in the book of Růžička [22]. A mathematical application is the study of variational integrals with non-standard growth, see the papers by Acerbi and Mingione $[1,2]$.

Sobolev capacity for fixed exponent spaces has found a great number of uses (e.g. the monographs by Evans and Gariepy [8] and Heinonen, Kilpeläinen, and Martio [14]). It was introduced into the study of variable exponent spaces in [12] and has been applied to the investigation of zero boundary values of Sobolev functions in [13]. In [12] we required

2000 Mathematics Subject Classification: 31B15, 46E35.

Key words and phrases: variable exponent Sobolev space, Sobolev capacity.

The paper is in final form and no version of it will be published elsewhere. 
the assumption $1<\operatorname{essinf} p \leq \operatorname{ess} \sup p<\infty$ of the variable exponent $p$ to guarantee that our set-function is indeed a Choquet capacity. This is unsatisfactory, since there is no reason to expect this condition to be of relevance in this context. In this paper we show that the lower inequality needs to hold only locally. In particular we show in Corollary 4.2 that if the exponent $p$ is continuous, then zero capacity sets enjoy the usual subadditivity property.

2. Sobolev $p(\cdot)$-capacity. We denote by $\mathbb{R}^{n}$ the Euclidean space of dimension $n \geq 2$. For $x \in \mathbb{R}^{n}$ and $r>0$ we denote the open ball with center $x$ and radius $r$ by $B(x, r)$ and by $B(r)$ an open ball with radius $r$ when the center is of no importance. We will next introduce variable exponent Lebesgue and Sobolev spaces in $\mathbb{R}^{n}$; note that we nevertheless use the standard definitions of the spaces $L^{p}(\Omega)$ and $W^{1, p}(\Omega)$ in the fixed exponent case $p \geq 1$ with open $\Omega \subset \mathbb{R}^{n}$.

Let $p: \mathbb{R}^{n} \rightarrow[1, \infty)$ be a measurable function (called the variable exponent on $\mathbb{R}^{n}$ ). Throughout this paper the function $p$ denotes a variable exponent; also, we define $p^{+}=$ $\operatorname{ess}_{\sup _{x \in \mathbb{R}^{n}}} p(x)$ and $p^{-}=\operatorname{essinf}_{x \in \mathbb{R}^{n}} p(x)$. We define the variable exponent Lebesgue space $L^{p(\cdot)}\left(\mathbb{R}^{n}\right)$ to consist of all measurable functions $u: \mathbb{R}^{n} \rightarrow \mathbb{R}$ such that $\varrho_{p(\cdot)}(\lambda u)=$ $\int_{\mathbb{R}^{n}}|\lambda u(x)|^{p(x)} d x<\infty$ for some $\lambda>0$. The function $\varrho_{p(\cdot)}: L^{p(\cdot)}\left(\mathbb{R}^{n}\right) \rightarrow[0, \infty)$ is called the modular of the space $L^{p(\cdot)}\left(\mathbb{R}^{n}\right)$. We define a norm, the so-called Luxemburg norm, on this space by the formula

$$
\|u\|_{p(\cdot)}=\inf \left\{\lambda>0: \varrho_{p(\cdot)}(u / \lambda) \leq 1\right\}
$$

The variable exponent Sobolev space $W^{1, p(\cdot)}\left(\mathbb{R}^{n}\right)$ is the space of measurable functions $u: \mathbb{R}^{n} \rightarrow \mathbb{R}$ such that $u$ and the absolute value of the distributional gradient $\nabla u=$ $\left(\partial_{1} u, \ldots, \partial_{n} u\right)$ are in $L^{p(\cdot)}\left(\mathbb{R}^{n}\right)$. The function $\varrho_{1, p(\cdot)}: W^{1, p(\cdot)}\left(\mathbb{R}^{n}\right) \rightarrow[0, \infty)$ is defined by $\varrho_{1, p(\cdot)}(u)=\varrho_{p(\cdot)}(u)+\varrho_{p(\cdot)}(|\nabla u|)$. The norm $\|u\|_{1, p(\cdot)}=\|u\|_{p(\cdot)}+\|\nabla u\|_{p(\cdot)}$ makes $W^{1, p(\cdot)}\left(\mathbb{R}^{n}\right)$ a Banach space. For more details on the variable exponent spaces we refer to $[17]$ or $[10]$.

We recall the definition and basic properties of the Sobolev $p(\cdot)$-capacity from $[12$, Section 3]. For $E \subset \mathbb{R}^{n}$ we denote

$$
S_{p(\cdot)}(E)=\left\{u \in W^{1, p(\cdot)}\left(\mathbb{R}^{n}\right): u \geq 1 \text { in an open set containing } E\right\} .
$$

The Sobolev $p(\cdot)$-capacity of $E$ is defined by

$$
C_{p(\cdot)}(E)=\inf _{u \in S_{p(\cdot)}(E)} \varrho_{1, p(\cdot)}(u)
$$

In case $S_{p(\cdot)}(E)=\emptyset$, we set $C_{p(\cdot)}(E)=\infty$. For arbitrary measurable exponents $p: \mathbb{R}^{n} \rightarrow$ $[1, \infty)$ the set function $E \mapsto C_{p(\cdot)}(E)$ has the following properties, [12, Theorem 3.1]:

(i) $C_{p(\cdot)}(\emptyset)=0$.

(ii) [Monotonicity] If $E_{1} \subset E_{2}$, then $C_{p(\cdot)}\left(E_{1}\right) \leq C_{p(\cdot)}\left(E_{2}\right)$.

(iii) If $E$ is a subset of $\mathbb{R}^{n}$, then

$$
C_{p(\cdot)}(E)=\inf _{\substack{E \subset U \\ U \text { open }}} C_{p(\cdot)}(U)
$$


(iv) If $E_{1}$ and $E_{2}$ are subsets of $\mathbb{R}^{n}$, then

$$
C_{p(\cdot)}\left(E_{1} \cup E_{2}\right)+C_{p(\cdot)}\left(E_{1} \cap E_{2}\right) \leq C_{p(\cdot)}\left(E_{1}\right)+C_{p(\cdot)}\left(E_{2}\right) .
$$

(v) If $K_{1} \supset K_{2} \supset \ldots$ are compact, then

$$
\lim _{i \rightarrow \infty} C_{p(\cdot)}\left(K_{i}\right)=C_{p(\cdot)}\left(\bigcap_{i=1}^{\infty} K_{i}\right) .
$$

If $1<p^{-} \leq p^{+}<\infty$, then the following additional properties hold, [12, Theorem 3.2]:

(vi) If $E_{1} \subset E_{2} \subset \ldots$ are subsets of $\mathbb{R}^{n}$, then

$$
\lim _{i \rightarrow \infty} C_{p(\cdot)}\left(E_{i}\right)=C_{p(\cdot)}\left(\bigcup_{i=1}^{\infty} E_{i}\right) .
$$

(vii) [Subadditivity] If $E_{i} \subset \mathbb{R}^{n}$ for $i=1,2, \ldots$, then

$$
C_{p(\cdot)}\left(\bigcup_{i=1}^{\infty} E_{i}\right) \leq \sum_{i=1}^{\infty} C_{p(\cdot)}\left(E_{i}\right) .
$$

This means that if $1<p^{-} \leq p^{+}<\infty$, then the set function $E \mapsto C_{p(\cdot)}(E), E \subset \mathbb{R}^{n}$, is a Choquet capacity, see [12, Corollary 3.4]. Note that if (vi) holds, then it and (iv) imply that (vii) holds as well. For the proof we refer to [12, Theorem 3.2].

3. Relaxing the condition $1<p^{-}$. We denote by $\chi_{G}$ the characteristic function of the set $G \subset \mathbb{R}^{n}$.

Proposition 3.1. Let $E \subset \mathbb{R}^{n}$. If $p^{+}<\infty$, then

$$
\lim _{R \rightarrow \infty} C_{p(\cdot)}(E \cap B(R))=C_{p(\cdot)}(E) .
$$

Proof. Since $E \cap B(R) \subset E$ for all $R$, it follows from (ii) that the right-hand side of (3.2) is greater than or equal to the left-hand side. To prove the opposite inequality, suppose first that $E$ is a set with finite $p(\cdot)$-capacity. Note that

$$
C_{p(\cdot)}(E \backslash B(R))+C_{p(\cdot)}(E \cap B(R)) \geq C_{p(\cdot)}(E)+C_{p(\cdot)}(\emptyset)=C_{p(\cdot)}(E)
$$

by property (iv). We will show that the first term on the left-hand side goes to zero as $R \rightarrow \infty$, from which the desired inequality follows.

Let us choose an open set $O$ containing $E$ and a function $u \in W^{1, p(\cdot)}\left(\mathbb{R}^{n}\right)$ with $u(x) \geq$ 1 for all $x \in O$ such that $\rho_{1, p(\cdot)}(u) \leq 2 C_{p(\cdot)}(E)$. It is clear that $\left\|\chi_{\mathbb{R}^{n} \backslash B(R)} u\right\|_{p(\cdot)} \rightarrow 0$ and $\left\|\chi_{\mathbb{R}^{n} \backslash B(R)} \nabla u\right\|_{p(\cdot)} \rightarrow 0$ as $R \rightarrow \infty$. For $R>2$ let $\phi$ be a 1-Lipschitz function with $0 \leq \phi \leq 1, \phi(x)=0$ for $x \in B(R)$ and $\phi(x)=1$ for $x \in \mathbb{R}^{n} \backslash B(2 R)$. It is easy to calculate that

$$
\|\phi u\|_{1, p(\cdot)} \leq 2\left\|\chi_{\mathbb{R}^{n} \backslash B(R)} u\right\|_{p(\cdot)}+\left\|\chi_{\mathbb{R}^{n} \backslash B(R)} \nabla u\right\|_{p(\cdot)},
$$

and so $\|\phi u\|_{1, p(\cdot)} \rightarrow 0$ as $R \rightarrow \infty$. It is clear that $\phi u$ is a function which is at least one on the open set $O \backslash \overline{B(2 R)}$ which contains $E \backslash \overline{B(2 R)}$, so that $\phi u$ is a test function for $C_{p(\cdot)}(E \backslash \overline{B(2 R)})$. Since $p^{+}<\infty$ the norm and the modular tend to zero simultaneously [17, Theorem 2.4], and it follows that $C_{p(\cdot)}(E \backslash B(R)) \rightarrow 0$, which completes the proof in the case that $C_{p(\cdot)}(E)<\infty$. 
Suppose then that $C_{p(\cdot)}(E)=\infty$ but that

$$
\lim _{R \rightarrow \infty} C_{p(\cdot)}(E \cap B(R))<\infty .
$$

Let $M>1$ be a finite upper bound of $C_{p(\cdot)}(E \cap B(R))$. We denote $A_{i}=B(i+1) \backslash \overline{B(i)}$ for $i=1,2, \ldots, A_{0}=B(1)$, and $C_{i}=C_{p(\cdot)}\left(E \cap A_{i}\right)$. Define the function $\phi_{i}: \mathbb{R}^{n} \rightarrow[0,1]$ by $\phi_{i}(x)=\phi(|x|-i)$ where

$$
\phi(s)= \begin{cases}1+s, & \text { for }-1 \leq s \leq 0 \\ 1, & \text { for } 0 \leq s \leq 1, \\ 2-s, & \text { for } 1 \leq s \leq 2, \\ 0, & \text { otherwise }\end{cases}
$$

Note that $\phi_{i}(x)$ is 1-Lipschitz.

Fix an integer $k \geq 0$ and $j=0,1,2$. Let $u \in S_{p(\cdot)}(E \cap B(3 k+j))$ be such that $\rho_{1, p(\cdot)}(u)<2 M$. Denote $A_{3 i+j}^{3}=A_{3 i+j-1} \cup A_{3 i+j} \cup A_{3 i+j+1}$. Since the support of $\phi_{3 i+j}$ lies in $A_{3 i+j}^{3}$, we have

$$
\begin{aligned}
\sum_{i=1}^{k} \rho_{1, p(\cdot)}\left(\phi_{3 i+j} u\right) & =\sum_{i=1}^{k} \int_{\mathbb{R}^{n}}\left|\phi_{3 i+j} u\right|^{p(x)}+\left|\nabla\left(\phi_{3 i+j} u\right)\right|^{p(x)} d x \\
& \leq \sum_{i=1}^{k} \int_{A_{3 i+j}^{3}} 2^{p^{+}}\left(|u|^{p(x)}+|\nabla u|^{p(x)}\right) d x \\
& =2^{p^{+}} \rho_{1, p(\cdot)}(u) \leq 2^{p^{+}+1} M .
\end{aligned}
$$

Since $u \phi_{3 i+j} \in S_{p(\cdot)}\left(E \cap A_{3 i+j}\right)$, this gives

$$
\sum_{i=1}^{k} C_{3 i+j} \leq \sum_{i=1}^{k} \rho_{1, p(\cdot)}\left(u \phi_{3 i+j}\right) \leq 2^{p^{+}+1} M .
$$

Adding these inequalities for $j=0,1,2$ gives $C_{1}+\ldots+C_{3 k} \leq 6 \cdot 2^{p^{+}} M$. Since the upper bound does not depend on $k$, we further get

$$
\sum_{i=1}^{\infty} C_{i} \leq 6 \cdot 2^{p^{+}} M
$$

Let us then choose for every integer $i \geq 0$ a function $u_{i} \in S_{p(\cdot)}\left(E \cap A_{i}\right)$ such that $\rho_{1, p(\cdot)}\left(u_{i}\right) \leq 2 C_{i}$. Then $u_{i} \phi_{i} \in S_{p(\cdot)}\left(E \cap A_{i}\right)$ and

$$
\rho_{1, p(\cdot)}\left(\phi u_{i}\right) \leq 2^{p^{+}} \rho_{1, p(\cdot)}\left(u_{i}\right) \text {. }
$$

It is easy to see that $w=\sum_{i=0}^{\infty} u_{i} \phi_{i}$ is in $S_{p(\cdot)}(E)$. With the convention $\phi_{-1} u_{-1} \equiv 0$ we find that

$$
\begin{aligned}
\rho_{1, p(\cdot)}(w)= & \sum_{i=0}^{\infty} \int_{A_{i}}\left|\phi_{i-1} u_{i-1}+\phi_{i} u_{i}+\phi_{i+1} u_{i+1}\right|^{p(x)} \\
& +\left|\nabla\left(\phi_{i-1} u_{i-1}+\phi_{i} u_{i}+\phi_{i+1} u_{i+1}\right)\right|^{p(x)} d x \\
\leq & 3^{p^{+}} \sum_{i=0}^{\infty} \rho_{1, p(\cdot)}\left(\phi u_{i}\right) \leq 6^{p^{+}} \sum_{i=0}^{\infty} C_{i} \leq 6 \cdot 12^{p^{+}} M<\infty
\end{aligned}
$$


contrary to the assumption that $C_{p(\cdot)}(E)=\infty$. This contradiction shows that the assumption $\lim _{R \rightarrow \infty} C_{p(\cdot)}(E \cap B(R))<\infty$ was false, which concludes the proof.

Lemma 3.3. Let $p^{+}<\infty$ and $E \subset B(1)$. Define $A_{r}=B(r+1) \backslash \overline{B(r)}$ and

$$
A_{r}(u)=\int_{A_{r}}|u|^{p(x)}+|\nabla u|^{p(x)} d x
$$

for $u \in W^{1, p(\cdot)}\left(\mathbb{R}^{n}\right)$. If $u \in S_{p(\cdot)}(E)$ and $\rho_{1, p(\cdot)}(u) \leq C_{p(\cdot)}(E)+\varepsilon$ for $\varepsilon>0$, then $A_{k}(u) \leq 2 \varepsilon$ for every

$$
k \geq 2^{2 p^{+}}\left(\frac{C_{p(\cdot)}(E)}{\varepsilon}+1\right)+2 .
$$

Proof. Define a Lipschitz function $\phi_{r}: \mathbb{R}^{n} \rightarrow[0,1]$ by

$$
\phi_{r}(x)= \begin{cases}1 & \text { for }|x| \leq r \\ 1-(|x|-r) & \text { for } r \leq|x| \leq r+1, \\ 0 & \text { for }|x| \geq r+1 .\end{cases}
$$

Fix $\varepsilon>0$ and let $u \in S_{p(\cdot)}(E)$ with $\rho_{1, p(\cdot)}(u) \leq C_{p(\cdot)}(E)+\varepsilon$. Then $u \phi_{r} \in S_{p(\cdot)}(E)$ for $r \geq 1$ and so $\rho_{1, p(\cdot)}\left(u \phi_{r}\right) \geq C_{p(\cdot)}(E) \geq \rho_{1, p(\cdot)}(u)-\varepsilon$. This gives

$$
\int_{A_{r}}\left|u \phi_{r}\right|^{p(x)}+\left|\nabla\left(u \phi_{r}\right)\right|^{p(x)} d x+\varepsilon \geq \int_{\mathbb{R}^{n} \backslash B(r)}|u|^{p(x)}+|\nabla u|^{p(x)} d x .
$$

Since $\left|u \phi_{r}\right| \leq|u|$ and $\left|\nabla\left(u \phi_{r}\right)\right| \leq\left|\phi_{r} \nabla u\right|+\left|u \nabla \phi_{r}\right| \leq|\nabla u|+|u|$, we further get

$$
\begin{aligned}
2^{p^{+}} \int_{A_{r}}|u|^{p(x)}+|\nabla u|^{p(x)} d x+\varepsilon & \geq \int_{A_{r}}|u|^{p(x)}+(|\nabla u|+|u|)^{p(x)} d x+\varepsilon \\
& \geq \int_{\mathbb{R}^{n} \backslash B(r)}|u|^{p(x)}+|\nabla u|^{p(x)} d x .
\end{aligned}
$$

Expressing this in terms of $A_{r}(u)$ gives

$$
2^{p^{+}} A_{r}(u)+\varepsilon \geq \sum_{i=r}^{\infty} A_{i}(u)
$$

Suppose then that $A_{k}(u) \geq 2 \varepsilon$. It follows from (3.4) that $2^{p^{+}} A_{i}(u)+\varepsilon \geq \sum_{j=i}^{\infty} A_{j}(u) \geq$ $A_{k}(u)$ for $i \leq k$. Using this in (3.4) gives

$$
2^{p^{+}} A_{1}(u)+\varepsilon \geq \sum_{i=1}^{\infty} A_{i}(u) \geq(k-1) \frac{A_{k}(u)-\varepsilon}{2^{p^{+}}}+A_{k}(u) \geq(k-1) \frac{\varepsilon}{2^{p^{+}}}+2 \varepsilon .
$$

Since $A_{1}(u) \leq \rho_{1, p(\cdot)}(u) \leq C_{p(\cdot)}(E)+\varepsilon$ this gives

$$
2^{p^{+}}\left(C_{p(\cdot)}(E)+\varepsilon\right) \geq(k-1) \frac{\varepsilon}{2^{p^{+}}},
$$

so that $2^{2 p^{+}}\left(C_{p(\cdot)}(E) / \varepsilon+1\right)+1 \geq k$. This means that if $2^{2 p^{+}}\left(C_{p(\cdot)}(E) / \varepsilon+1\right)+2 \leq k$ then $A_{k}(u) \leq 2 \varepsilon$, which was to be shown.

We say that $p$ is locally bounded away from 1 if $\operatorname{ess~} \inf _{x \in K} p(x)>1$ for every compact $K \subset \mathbb{R}^{n}$. 
THEOREM 3.5. Let $E_{i}$ be a sequence of increasing sets. Suppose that $p^{+}<\infty$ and $p$ is locally bounded away from 1 . Then the capacity has property (vi).

Proof. Let us denote $E=\bigcup_{i=1}^{\infty} E_{i}$. It follows from property (ii) that

$$
\lim _{i \rightarrow \infty} C_{p(\cdot)}\left(E_{i}\right) \leq C_{p(\cdot)}(E) .
$$

It remains to prove the opposite inequality. It suffices to consider the case where $C_{p(\cdot)}\left(E_{i}\right)$ $<M<\infty$ for every $i$.

Let us first assume that $E$ is bounded. We choose the coordinate system so that $E \subset B(1)$. Fix $\varepsilon>0$ and choose $u_{i} \in S_{p(\cdot)}\left(E_{i}\right)$ such that $\rho_{1, p(\cdot)}\left(u_{i}\right) \leq C_{p(\cdot)}\left(E_{i}\right)+\varepsilon<M+\varepsilon$ for every $i$. Let

$$
k=2^{2 p^{+}}\left(\frac{M}{\varepsilon}+1\right)+2
$$

and let the operators $A_{i}$ be defined as in Lemma 3.3. It follows from the lemma that $A_{k}\left(u_{i}\right) \leq 2 \varepsilon$ for every $i$. Define $\phi: \mathbb{R}^{n} \rightarrow[0,1]$ by

$$
\phi(x)= \begin{cases}1 & \text { for }|x| \leq k \\ 1-(|x|-k) & \text { for } k \leq|x| \leq k+1 \\ 0 & \text { for }|x| \geq k+1\end{cases}
$$

Since $\operatorname{spt} \phi=B(k+1)$, we have

$$
\rho_{1, p(\cdot)}\left(\phi u_{i}\right)=\sum_{j=0}^{k-1} A_{j}\left(u_{i}\right)+A_{k}\left(\phi u_{i}\right)
$$

As in the proof of Lemma 3.3, we find that $A_{k}\left(\phi u_{i}\right) \leq 2^{p^{+}} A_{k}\left(u_{i}\right)$, and since $A_{k}\left(u_{i}\right) \leq 2 \varepsilon$ we get

$$
\rho_{1, p(\cdot)}\left(\phi u_{i}\right) \leq \sum_{j=0}^{k-1} A_{j}\left(u_{i}\right)+2^{p^{+}+1} \varepsilon \leq \rho_{1, p(\cdot)}\left(u_{i}\right)+2^{p^{+}+1} \varepsilon \leq C_{p(\cdot)}\left(E_{i}\right)+\left(2^{p^{+}+1}+1\right) \varepsilon .
$$

Let us define $q_{0}=\inf _{y \in B(k+1)} p(y)$ and a new variable exponent by $q(x)=$ $\max \left\{p(x), q_{0}\right\}$ for every $x \in \mathbb{R}^{n}$. It is clear that $\phi u_{i} \in W^{1, q(\cdot)}\left(\mathbb{R}^{n}\right)$ for all $i$, and so we can use the reflexivity of the space $W^{1, q(\cdot)}\left(\mathbb{R}^{n}\right)$ and proceed as in the proof of Theorem 3.2, [12] and find a sequence of functions $w_{j}$ in $W^{1, q(\cdot)}\left(\mathbb{R}^{n}\right)$ each of which equals 1 in an open set containing $E$ with $\rho_{1, p(\cdot)}\left(w_{j}\right) \rightarrow \rho_{1, p(\cdot)}\left(\phi u_{i}\right)$ as $j \rightarrow \infty$. Since every $w_{j}$ is the sum of $\phi u_{i}$ 's, it is also easy to see that every $w_{j}$ is supported in $B(k+1)$. Therefore we may use the $w_{j}$ as test functions for the set $E$ in the space $W^{1, p(\cdot)}\left(\mathbb{R}^{n}\right)$, as well, and we conclude that

$$
C_{p(\cdot)}(E) \leq \lim _{i \rightarrow \infty} C_{p(\cdot)}\left(E_{i}\right)+\left(2^{p^{+}+1}+1\right) \varepsilon .
$$

Taking $\varepsilon \rightarrow 0$ completes the proof in the case of a bounded set $E$.

Suppose then that $E$ is unbounded. By what was just proved we have

$$
\lim _{i \rightarrow \infty} C_{p(\cdot)}\left(E_{i} \cap B(r)\right)=C_{p(\cdot)}(E \cap B(r))
$$

for every $r>0$. But it follows from Proposition 3.1 that

$$
\lim _{i \rightarrow \infty} C_{p(\cdot)}\left(E_{i}\right)=\lim _{i \rightarrow \infty} \lim _{r \rightarrow \infty} C_{p(\cdot)}\left(E_{i} \cap B(r)\right)=\lim _{r \rightarrow \infty} C_{p(\cdot)}(E \cap B(r))=C_{p(\cdot)}(E),
$$


where the swap of limit-taking order is permissible in the second equality since $(i, r) \mapsto$ $C_{p(\cdot)}\left(E_{i} \cap B(r)\right)$ is increasing in both variables. This completes the proof of the unbounded case.

REMARK 3.6. If $p$ is continuous, then it is locally bounded away from 1 if and only if it is strictly greater than 1 .

The previous theorem allows us to improve our result from [12, Corollary 3.4].

Corollary 3.7. Suppose that $p$ is bounded and locally bounded away from 1. Then $C_{p(\cdot)}(\cdot)$ is a Choquet capacity and an outer measure.

4. Zero capacity sets. Sets of zero capacity are of particular importance when dealing with any capacity. For instance it was shown in [13, Theorem 3.9] that zero boundary value spaces defined on $\Omega$ and $\Omega \backslash N$ concur if and only if $N$ has zero capacity.

In this section we prove that the regularity properties from the previous section hold for capacity zero sets under much fewer conditions than for arbitrary sets.

THEOREM 4.1. Suppose that $p$ is locally bounded and that $E_{i} \subset \mathbb{R}^{n}$ are zero capacity sets for $i \in \mathbb{N}$. Then $C_{p(\cdot)}\left(\cup E_{i}\right)=0$.

Proof. Let us denote $E=\cup E_{i}$ and define $p_{R}=\sup _{x \in B(R+2)} p(x)$.

Suppose first that $E$ is bounded, say $E \subset B(R)$. Let $\phi$ be an 1-Lipschitz continuous cut-off function supported in $B(2 R)$ with $\left.\phi\right|_{B(R)}=1$. Fix a positive $\varepsilon<2^{-p_{2 R}}$. For every $i$ choose a function $u_{i} \in S_{p(\cdot)}\left(E_{i}\right)$ such that $\rho_{1, p(\cdot)}\left(u_{i}\right) \leq \varepsilon 2^{-i}$. Since the function $\phi u_{i}$ is supported in $B(2 R)$ it is clear that the values of the exponent $p$ outside $B(2 R)$ do not effect $\rho_{1, p(\cdot)}\left(\phi u_{i}\right)$. Therefore we conclude that $\rho_{1, p(\cdot)}\left(\phi u_{i}\right) \leq 2^{p_{2 R}} \rho_{1, p(\cdot)}\left(u_{i}\right) \leq \varepsilon 2^{p_{2 R}-i}$. It follows from [10, Theorem 1.3] (since we need not consider $B(2 R)^{c}$ ) that

$$
\left\|\phi u_{i}\right\|_{1, p(\cdot)} \leq \rho_{1, p(\cdot)}\left(\phi u_{i}\right)^{1 / p_{2 R}} \leq 2 \varepsilon^{1 / p_{2 R}} 2^{-i / p_{2 R}} .
$$

Thus, by the triangle inequality,

$$
\left\|\phi \sum_{i=1}^{\infty} u_{i}\right\|_{1, p(\cdot)} \leq 2 \varepsilon^{1 / p_{2 R}} \sum_{i=1}^{\infty} 2^{-i / p_{2 R}}=2 \varepsilon^{1 / p_{2 R}} \frac{1}{1-2^{-1 / p_{2 R}}} .
$$

Since $\phi \sum_{i=1}^{\infty} u_{i} \in S_{p(\cdot)}(E)$ it follows that

$$
C_{p(\cdot)}(E) \leq \frac{2 \varepsilon^{1 / p_{2 R}}}{1-2^{-1 / p_{2 R}}},
$$

and so $C_{p(\cdot)}(E)=0$, since $\varepsilon$ can be arbitrarily small.

Suppose then that $E$ is unbounded. By monotonicity it follows that $C_{p(\cdot)}\left(E_{i} \cap B(R)\right)=$ 0 for any $R>0$. Thus, it follows from the first part of the proof that $C_{p(\cdot)}(E \cap B(R))=0$ for any $R>0$. Next we show that this implies that $C_{p(\cdot)}(E)=0$. Let us denote $A_{R}^{*}=$ $B(R+2) \backslash \bar{B}(R-1)$ and $A_{R}=B(R+1) \backslash \bar{B}(R)$.

By monotonicity of the capacity we have $C_{p(\cdot)}\left(E \cap A_{R}\right)=0$ for every $R>0$. Let us choose for every positive integer $i$ a function $u_{i} \in S_{p(\cdot)}\left(E \cap A_{i}\right)$ such that $\rho_{1, p(\cdot)}\left(u_{i}\right) \leq$ $6^{-p_{R}} \varepsilon 2^{-i}$. Let $\phi_{i}$ denote a 1-Lipschitz function supported in $\overline{A_{i}^{*}}$ with $\left.\phi_{i}\right|_{A_{i}}=1$. We find 
that

$$
\begin{aligned}
\rho_{1, p(\cdot)}\left(\sum_{i=0}^{\infty} \phi_{i} u_{i}\right) \leq & \sum_{i=0}^{\infty} \int_{A_{i}}\left|u_{i-1}+u_{i}+u_{i+1}\right|^{p(x)} \\
& +\left|u_{i-1}+u_{i}+u_{i+1}+\nabla\left(u_{i-1}+u_{i}+u_{i+1}\right)\right|^{p(x)} d x \\
\leq & \sum_{i=0}^{\infty} \int_{A_{i}}\left(3^{p_{R}-1}+6^{p_{R}-1}\right)\left(\left|u_{i-1}\right|^{p(x)}+\left|u_{i}\right|^{p(x)}+\left|u_{i+1}\right|^{p(x)}\right) \\
& +6^{p_{R}-1}\left(\left|\nabla u_{i-1}\right|^{p(x)}+\left|\nabla u_{i}\right|^{p(x)}+\left|\nabla u_{i+1}\right|^{p(x)}\right) d x \\
\leq & 6^{p_{R}} \sum_{i=0}^{\infty} \rho_{1, p(\cdot)}\left(u_{i}\right) \leq \sum_{i=0}^{\infty} \varepsilon 2^{-i}=\varepsilon
\end{aligned}
$$

Since $\sum_{i=0}^{\infty} \phi_{i} u_{i} \in S_{p(\cdot)}(E)$, this means that $C_{p(\cdot)}(E)=0$, as was to be shown.

COROLlary 4.2. Suppose that the variable exponent $p$ is continuous. Then the countable union of zero $p(\cdot)$-capacity sets has $p(\cdot)$-capacity equal to zero, as well.

\section{References}

[1] E. Acerbi and G. Mingione, Regularity results for a class of functionals with non-standard growth, Arch. Ration. Mech. Anal. 156 (2001), 121-140.

[2] E. Acerbi and G. Mingione, Regularity results for stationary electro-rheological fluids, Arch. Ration. Mech. Anal. 164 (2002), 213-259.

[3] D. Cruz-Uribe, A. Fiorenze and C. J. Neugebauer, The maximal function on variable $L^{p}$ spaces, Ann. Acad. Sci. Fenn. Math. 28 (2003), 223-238; 29 (2004), 247-249.

[4] L. Diening, Maximal function on generalized Lebesgue spaces $L^{p(\cdot)}$, Math. Inequal. Appl. 7 (2004), 245-254.

[5] L. Diening, Riesz Potential and Sobolev Embeddings of generalized Lebesgue and Sobolev Spaces $L^{p(\cdot)}$ and $W^{k, p(\cdot)}$, Math. Nachr. 263 (2004), 31-43.

[6] D. E. Edmunds and J. Rákosník, Sobolev embedding with variable exponent, Studia Math. 143 (2000), 267-293.

[7] D. E. Edmunds and J. Rákosník, Sobolev embedding with variable exponent, II, Math. Nachr. 246-247 (2002), 53-67.

[8] L. C. Evans and R. F. Gariepy, Measure Theory and Fine Properties of Functions, Studies in Advanced Mathematics, CRC Press, Boca Raton, 1992.

[9] X. Fan, J. Shen, and D. Zhao, Sobolev embedding theorems for spaces $W^{k, p(x)}$, J. Math. Anal. Appl. 262 (2001), 749-760.

[10] X. Fan and D. Zhao, On the spaces $L^{p(x)}(\Omega)$ and $W^{k, p(x)}(\Omega)$, J. Math. Anal. Appl. 263 (2001), 424-446.

[11] P. Harjulehto and P. Hästö, A capacity approach to the Poincaré inequality and Sobolev imbedding in variable exponent Sobolev space, Rev. Mat. Complut. 17 (2004), 129-146.

[12] P. Harjulehto, P. Hästö, M. Koskenoja, and S. Varonen, Sobolev capacity on the space $W^{1, p(\cdot)}\left(\mathbb{R}^{n}\right)$, J. Function Spaces Appl. 1 (2003), 17-33.

[13] P. Harjulehto, P. Hästö, M. Koskenoja and S. Varonen, Dirichlet energy integral and variable exponent Sobolev spaces with zero boundary values, Reports of the Department of Mathematics, Preprint 353, April 2003. 
[14] J. Heinonen, T. Kilpeläinen and O. Martio, Nonlinear Potential Theory of Degenerate Elliptic Equations, Oxford Mathematical Monographs, Oxford University Press, Oxford, 1993.

[15] H. Hudzik, The problems of separability, duality, reflexivity and of comparison for generalized Orlicz-Sobolev spaces $W_{M}^{k}(\Omega)$, Comment. Math. Prace Mat. 21 (1980), 315-324.

[16] V. Kokilasvili and S. Samko, Maximal and fractional operators in weighted $L^{p(x)}$ spaces, Rev. Mat. Iberoamericana 20 (2004), 493-515.

[17] O. Kováčik and J. Rákosník, On spaces $L^{p(x)}$ and $W^{1, p(x)}$, Czechoslovak Math. J. 41 (116) (1991), 592-618.

[18] J. Musielak, Orlicz Spaces and Modular Spaces, Springer-Verlag, Berlin, 1983.

[19] A. Nekvinda, Equivalence of $l^{\left\{p_{n}\right\}}$ norms and shift operators, Math. Inequal. Appl. 5 (2002), 711-723.

[20] W. Orlicz, Über konjugierte Exponentenfolgen, Studia Math. 3 (1931), 200-212.

[21] L. Pick and M. Růžička, An example of a space $L^{p(x)}$ on which the Hardy-Littlewood maximal operator is not bounded, Expo. Math. 19 (2001), 369-371.

[22] M. Růžička, Electrorheological Fluids, Modeling and Mathematical Theory, SpringerVerlag, Berlin, 2000.

[23] I. I. Sharapudinov, On the topology of the space $L^{p(t)}([0 ; 1])$, Math. Notes 26 (1979), 796-806. [Translation of Mat. Zametki 26 (1978), 613-632.] 